\title{
Kopfschmerzen durch Pharmapreise
}

\author{
Unikliniken klagen über einen rasanten Anstieg der Preise für patentgeschützte \\ Medikamente. Offenbar nutzen Pharmafirmen den stationären Markt, um ihre \\ Verluste durch Rabattierungen im ambulanten Bereich auszugleichen.
}

$\square$ igentlich ist Holger Knoth ein Freund starker Worte. Als Leiter der Klinikapotheke der Uniklinik Dresden wählt der Sachse seine Worte üblicherweise vorsichtig. Manchmal aber kann auch er ungewohnt deutlich werden. Etwa, wenn man ihn auf Yervoy anspricht. Das Immunantikörperpräparat von Bristol-Myers Squibb gilt seit seiner Einführung im vergangenen Jahr als wichtiges Medikament in der Behandlung der besonders bösartigen Hautkrebsvariante Malignes Melanom. Doch den Erfolg lässt sich Bristol Myers Squibb fürstlich bezahlen. Für die Initialbehandlung mit nur vier Anwendungen kassiert das US-Pharmaunternehmen stolze 101.000 Euro. „Dieser Preis ist jenseits von Gut und Böse“, kritisiert der Dresdner Uniapotheker.

Yervoy ist ein Extrembeispiel für eine Entwicklung, die vor allem den forschenden Unikliniken Kopfschmerzen bereitet. Sie vor allem setzen neue und innovative Präparate ein. Seit Jahren steigen aber die Kosten für patentgeschützte Medikamente speziell in den Bereichen Onkologie, Hämatologie und Rheumatologie rasant, während die Kosten im restlichen Klinikarzneimittelmarkt durch verstärkten Generikaeinsatz nur noch leicht wachsen.
Dieser Trend könnte sich nach Befürchtungen des Verbands der Universitätsklinika Deutschlands (VUD) verstärken, obwohl der Gesetzgeber mit dem neuen Arzneimittelneuordnungsgesetz (AMNOG) hohen Medikamentenpreisen einen Riegel vorschieben wollte. „Die Befürchtung ist leider gut begründet“, warnt Ralf Heyder vom VUD.

Das Problem hoher Preise bei patentgeschützten Medikamenten ist kein neu-

vorzuschreiben, dass die Pharmafirmen dann auch den Kliniken einen entsprechenden Herstellerrabatt wie im ambulanten GKV-Markt einräumen müssen. Im Verhältnis zwischen Hersteller und Krankenhaus sollte weiter die freie Preisbildung gelten.

Die Folgen der Novelle beschrieb Georg Baum, Hauptgeschäftsführer der Deutschen Krankenhausgesellschaft (DKG), im Mai 2010 bei einer An-

\section{„Wenn sich die Industrie mit der Geheimhaltung der Rabattpreise durchsetzt, wird die Asymmetrie bei den Arzneimittelpreisen zwischen Kliniken und am- bulantem GKV-Markt weiter zunehmen.“}

Frank Dörje, Arbeitsgruppe der Leitenden Apotheker der Universitätsklinika (LAUD)

es Phänomen, verschärft sich aber seit 2009. Damals trat die 15. Novelle des Arzneimittelgesetzes (AMG) in Kraft, mit der die Bundesregierung unter anderem versuchte, den Kostenanstieg bei den Arzneimitteln zu dämpfen. Durch die Novelle wurde den Krankenkassen bei Arzneimitteln eine Rabattierung von sechs Prozent gegenüber den Kliniken eingeräumt. Ohne allerdings zwingend hörung im Gesundheitsausschuss des Bundestags. „Seit der 15. AMGNovelle hat die pharmazeutische Industrie massive Preiserhöhungen bei den Zytostatika vorgenommen." Was der DKG-Hauptgeschäftsführer da im Rückblick erklärte, war zugleich schon ein Versuch, weiteres Unheil abzuwenden. In jener besagten Anhörung ging es um den Entwurf des 


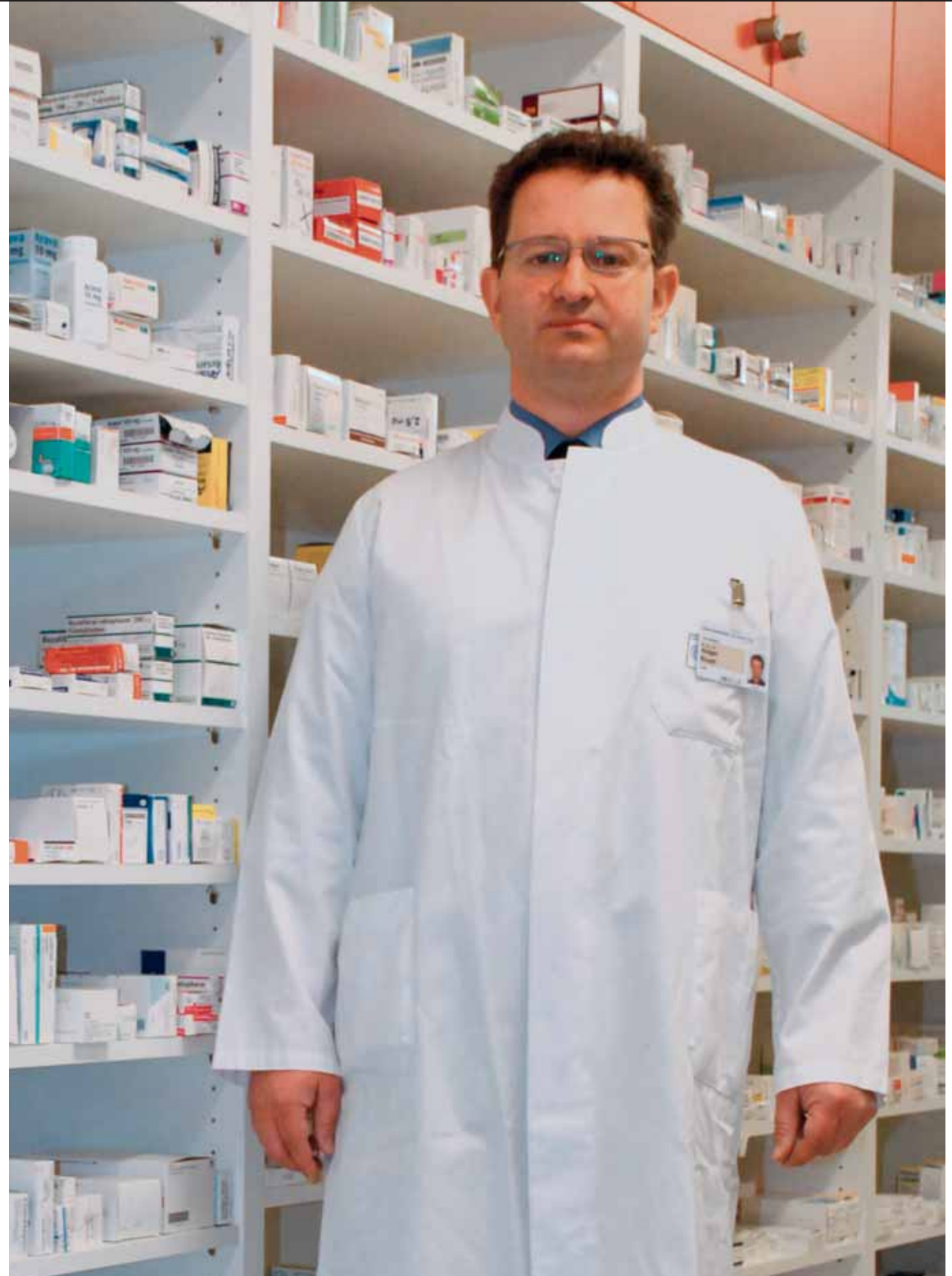

Holger Knoth: „Jenseits von Gut und Böse“ nennt der Chef-Apotheker der Uniklinik Dresden den Preis für das patentgeschützte Krebsmedikament Yervoy. Eine Initialbehandlung mit vier Anwendungen kostet 101.000 Euro.

\section{Fast zehn Prozent Preissteigerung}

Wie sehr die Schere mittlerweile zwischen allgemeinen Klinikarzneimitteln und dem Markt für spezielle patentgeschützte Medikamente auseinandergeht, zeigt erst ein genauerer Blick auf die aktuellen Zahlen. So stieg laut dem Pharmamarktforschungsunternehmen IMS Health der Gesamtumsatz für Arzneimittel in den Kliniken im vergangenen Jahr nur noch um 1,3 Prozent auf 4,23 Milliarden Euro. Betrachtet man die Zahlen für patentgeschützte Medikamente im gesamten GKV-Markt, liegt das Wachstum bereits bei 4,1 Prozent.

In den Kliniken jedoch stiegen deren Preise laut IMS binnen einen Jahres um durchschnittlich 9 Prozent. Das Ende der Fahnenstange ist damit noch lange nicht erreicht, denn die bereits beschriebenen besonders innovativen Medikamente bei der Krebs- und Rheumabehandlung legten - je nach Quelle - um mehr als 20 Prozent zu (siehe Tabelle auf Seite 32).
GKV-Änderungsgesetzes, welches drei Monate später in Kraft treten sollte. Das Gesetz verschärfte die Situation erneut, weil es im ambulanten Sektor den Rabatt auf 16 Prozent erhöhte und ein rückwirkendes Preismoratorium für patentgeschützte Medikamente festlegte. Eine Festlegung auf einen Herstellerrabatt gegenüber den Kliniken unterblieb erneut, „weil das politisch so gewollt ist", sagt Holger Knoth.

\section{VUD und DKG fordern Preisdeckel}

Schon damals orakelte Frank Dörje, Chef der Uniklinik-Apotheke Erlangen und Sprecher der Arbeitsgruppe der Leitenden Apotheker der Universitätsklinika Deutschlands (LAUD), dass dieses Preisgefälle die ambulante Weiterversorgung der Kliniken - etwa für Krebspatienten - gefährden könne, weil sie im ambulanten Bereich keine ähnlich guten Preise wie im geregelten ambulanten Markt anbieten könnten. Parallel dazu werde es im stationären Bereich zu „erheblichen Kostenbelastungen“ kommen, so Dörje. Darum fordern DKG, VUD und Uni-Apotheker seitdem vehement einen Preisdeckel für Arzneimittel in den Kliniken.

Knapp zwei Jahre nach dieser Anhörung sind Dörjes Befürchtungen teilweise eingetreten. „Die Asymmetrie bei der Preisbildung zwischen Klinik und ambulantem Markt besteht weiter. Die Hersteller haben quasi über Nacht noch einmal zehn Prozent ihres Erlöses verloren und versuchen, die Verluste im ambulanten GKV-Markt im stationären Bereich zu kompensieren“, sagt Dörje. Viele Firmen beharren auf Listenpreise in den Kliniken, weil sie sonst angeblich „Pleite gehen würden“, schildert Frank Dörje die Klagen der Unternehmen aus den Preisverhandlungen.

Wie diese Asymmetrie bei der Preisbildung wirkt, schildert Torsten Hoppe-Tichy, Leiter der Heidelberger 
Preisvergleich zwischen Stationär und Ambulant

\begin{tabular}{l|l|r|r|r}
\hline Präparat & $\begin{array}{l}\text { Herstellererlös } \\
\text { ab }\end{array}$ & GKV ambulant** & $\begin{array}{l}\text { Einkaufpreis Kran- } \\
\text { kenhaus stationär* }\end{array}$ & $\begin{array}{c}\text { Differenz stationär- } \\
\text { ambulant }\end{array}$ \\
\hline Avastin $\mathbf{4 0 0 ~ m g ~}$ & 1.1 .2007 & $1.211,66$ Euro & $1.192,33$ Euro & $-1,6 \%$ \\
\hline & 1.9 .2009 & $1.246,80$ Euro & $1.293,50$ Euro & $3,7 \%$ \\
\hline & 1.8 .2010 & $1.076,78$ Euro & $1.328,46$ Euro & $23,4 \%$ \\
\hline Herceptin 150 mg & 1.1 .2007 & 618,07 Euro & 608,21 Euro & $-1,6 \%$ \\
\hline & 1.9 .2009 & 635,99 Euro & 663,40 Euro & $4,3 \%$ \\
\hline & 1.8 .2010 & 549,27 Euro & 681,33 Euro & $24 \%$ \\
\hline & 1.1 .2007 & 576,64 Euro & 567,44 Euro & $-1,6 \%$ \\
\hline & 1.9 .2009 & 616,50 Euro & 643,06 Euro & $4,3 \%$ \\
\hline & 1.8 .2010 & 532,43 Euro & 660,44 Euro & $24 \%$ \\
\hline
\end{tabular}

* Basis: Erhebung der Einkaufspreise von fünf Universitätsklinika

** Preise ab 1.8.2010 inklusive Abschlag infolge Preismoratorium und höherem Herstellerrabatt

Unklare Preisgestaltung: Kliniken müssen für Antikörper-Präparate deutlich mehr zahlen als der ambulante Bereich. Besonders deutlich ist der Preisunterschied mit über 250 Euro pro Dosis bei dem Krebsmedikament Avastin.

Uniklinik-Apotheke und 1. Vizepräsident des Bundesverbands Deutscher Krankenhausapotheker (ADKA). Da Krankenhausapotheken bei der ambulanten Abrechnung nicht teurer sein dürfen als private Apotheken, werden ihnen bei Abrechnung des Rezepts von den Kassen 16 Prozent Rabatt abgezogen. Die Hersteller müssen jedoch nur der Krankenkasse den Rabatt gewähren. „Wenn dann der Einkaufspreis des Krankenhauses höher ist als der Abrechnungspreis minus 16 Prozent, haben die Krankenhäuser ein Problem“, so Hoppe-Tichy. Beharrt ein Lieferant dann auf den Listenpreisen, ist Kurioses möglich. „Mir haben einige Kollegen berichtet, dass den Krankenhäusern nichts anderes übrig bleibt, als die Arzneimittel auf einem Rezept an eine private Apotheke zu verordnen.“

Dass bislang die schlimmsten Befürchtungen noch nicht eingetreten sind, hat mehrere Gründe: Die Kostensenkungen bei anderen Arzneimittelgruppen sorgen noch dafür, dass die Mehrkosten bei den patentgeschützten Medikamenten teilweise abgemildert werden. Außeredem reagieren Pharmafirmen ganz unter- schiedlich in den Preisverhandlungen, wie Frank Dörje berichtet. Während etwa Pharmariesen wie Roche Krankenhäuser mit einer breiten Palette an Therapeutika beliefern und entsprechend flexibler agieren können, sind kleinere Firmen teilweise nur mit einem Medikament vertreten. Für diese wären 16 Prozent Zwangsrabatt ein wirtschaftliches Problem. Dörje: „Es gibt daher Unternehmen, die gar keinen Rabatt einräumen, Firmen, die teilweise nachlassen bis hin zu jenen, die den kompletten Rabatt einräumen." Die Gesamttendenz ist aber klar: „Die Hersteller haben ein extremes Interesse daran, die Preise hoch zu halten“, sagt Holger Knoth.

\section{vfa verweist auf „eigene Wege im Wettbewerb“}

Beim Verband der forschenden Arzneimittelhersteller (vfa) weiß man angeblich nichts von den Klagen der Kliniken über die Preisentwicklung bei patentgeschützten Medikamenten und die Kompensationsgeschäfte der Branche. Dabei saß 2010 die damalige Hauptgeschäftsführerin des vfa - Cornelia Yzer - neben Frank Dörje in der Bundestagsanhörung. Über die
Preispolitik sagt vfa-Sprecher Jochen Stemmler nur soviel: „Die Pharmaunternehmen stehen im Wettbewerb. Sie gehen daher im Markt eigene Wege, um ihre Interessen zu verfolgen.“

Wie sich die Situation zukünftig für die Kliniken entwickeln wird, hängt nicht unwesentlich von der laufenden Umsetzung des Arzneimittelneuordnungsgesetzes (AMNOG) ab, das seit 1. Januar 2011 in Kraft ist. Das AMNOG soll den Kostenanstieg bei Medikamenten begrenzen und sieht dafür ein Verfahren zur sogenannten Zusatznutzenbewertung durch den Gemeinsamen Bundesausschuss (GBA) vor. Wird in diesem Bewertungsverfahren kein Zusatznutzen bei nach dem 1.1.2011 eingeführten Medikamenten festgestellt, wird für das Medikament eine Festpreisgruppe bestimmt. Gibt es einen Zusatznutzen, handeln GKVSpitzenverband und Hersteller einen Rabatt zum Listenpreis aus. Sollte es keine Einigung geben, entscheidet eine Schiedsstelle in festgelegter Zeit. Spätestens zwölf Monate nach Markteinführung kommt es somit zu einer Preisfestsetzung.

\section{Es gibt keine Preistransparenz}

Die Umsetzung des AMNOG hinkt dem Zeitplan hinterher, weil sich Krankenkassen und Pharmahersteller lange nicht auf einen Länderkorb zur Festsetzung eines europäischen Vergleichspreises einigen konnten. Die Zusammensetzung ist wichtig für beide Seiten. Je mehr Länder mit höherem Preisniveau im Länderkorb berücksichtigt werden, desto besser für die Industrie. Der Streit um den Korb wurde Anfang März durch einen Spruch der Schiedsstelle beigelegt, der im Länderkorb 15 europäische Länder mit unterschiedlichen Preisniveaus berücksichtigt. Laut GKV-Spitzenverband sollen in diesem Jahr nun für mehr als 20 Wirkstoffe die Rabatte festgelegt werden. 


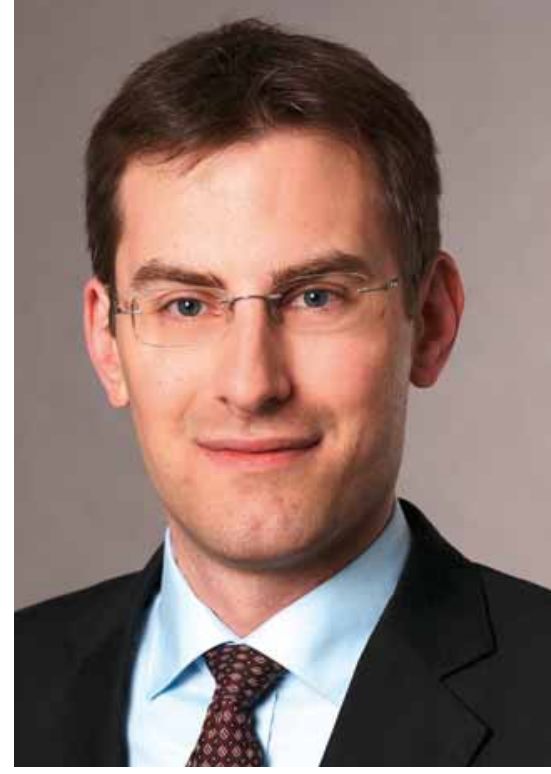

VUD-Referent Heyder: „Die Preissteigerungen bei den patentgeschützten Präparaten könnten sich trotz AMNOG weiter verstärken", sagt der künftige Generalsekretär des Verbands der Universitätsklinika.
Was zunächst gut klingt, hat für die Kliniken allerdings gleich mehrere Haken. Zum einen sind die Pharmafirmen auch nach dem AMNOG in den ersten zwölf Monaten nach Markteinführung ,weiterhin völlig frei in der Preisgestaltung“, sagt Frank Dörje. Zum anderen bleibt die Frage, ob die Rabattierung dieses Mal nun auch im stationären Bereich greifen soll. Beim VUD beobachtet man mit Sorge, dass die Pharmaindustrie derzeit daraufhin arbeitet, „die Ergebnisse der Rabattverhandlungen für geheim zu erklären. Hier darf keine Black Box für die Krankenhäuser entstehen“, kritisiert Ralf Heyder. Die Befürchtung: Bleiben die Rabattpreise geheim, könnten den Kliniken wieder mit höheren Listenpreise den Kürzeren ziehen.
Transparenz schafft Herstellern Preisprobleme in anderen Ländern Auf Seiten der Industrie hat man dagegen überhaupt kein Interesse, für Transparenz bei den Preisen zu sorgen. Denn: Deutschland ist Referenzland für den europäischen Arzneimittelmarkt. Würden die neuen Rabattpreise öffentlich, hätte die Pharmaindustrie in anderen europäischen Ländern ein erhebliches Preisproblem. Setzt sich jedoch die Industrie mit der Geheimhaltung durch, befürchtet Frank Dörje einen weiteren Kostenanstieg für die Kliniken. „In diesem Fall wird die Asymmetrie bei den Arzneimittelpreisen zwischen Kliniken und ambulantem GKV-Markt weiter zunehmen.“ घ

Guntram Doelfs 홍

\section{health. care. vitality.}

\section{Planung und Errichtung}

- General- und Totalübernehmerschaften, ÖPP

- Ziel- und Masterplanung

- Betriebsorganisationsplanung

- General- und Fachplanungen (MT, IT, TGA)

- Finanzierungssicherung

- Inbetriebnahme

- Sanierungsmanagement
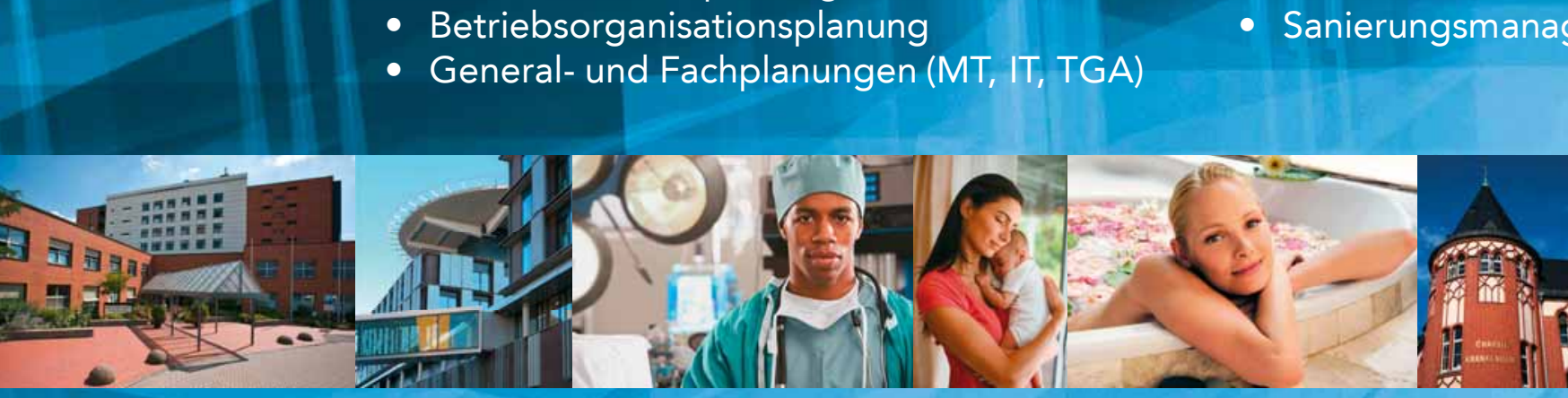

\section{Technische Betriebsführung}

- Medizintechnik

- Krankenhausbetriebstechnik

- Informationstechnik
- Sterilgutversorgung

- OP-Unit

- Finanzierung 